Supporting Information for

\title{
Synthesis and Characterization of Water-Soluble Conjugated Oligoelectrolytes for Near-Infrared Fluorescence Biological Imaging
}

Shin-Jae Woo ${ }^{\dagger}$, Sungmin Park ${ }^{\dagger}$, Ji-Eun Jeong ${ }^{\ddagger}$, Yoochan Hong ${ }^{\S}$, Minhee Ku ${ }^{\S}$,", Bo Yun Kim ${ }^{\perp}, I l^{\prime}$ Ho Jang ${ }^{\perp}$, Soon Chul Heo ${ }^{\perp}$, Taejun Wang ${ }^{n}$, Ki Hean Kimn, Jaemoon Yang*\&, ", Jae Ho Kim*, $\perp$ and Han Young Woo*t

${ }^{\dagger}$ Department of Cogno-Mechatronics Engineering, Pusan National University, Miryang, 50463, Republic of Korea

Department of Chemistry, Korea University, Seoul, 02841, Republic of Korea. E-mail: hywoo@korea.ac.kr (H. Y. Woo)

${ }^{\S}$ Department of Radiology, College of Medicine, Yonsei University, YUHS-KRIBB Medical Convergence Research Institute, Seoul, 03722, Republic of Korea. E-mail: 177hum@yuhs.ac (J. Yang)

"Brain Korea 21 PLUS Project for Medical Science, Yonsei University, Seoul, 03722, Republic of Korea

${ }^{\perp}$ Department of Physiology, School of Medicine, Pusan National University, Yangsan, 50612, Republic of Korea. E-mail: jhkimst@pusan.ac.kr (J. H. Kim)

${ }^{n}$ Division of Integrative Biosciences and Biotechnology, Department of Mechanical Engineering, Pohang University of Science and Technology, Pohang, 37673, Republic of Korea 

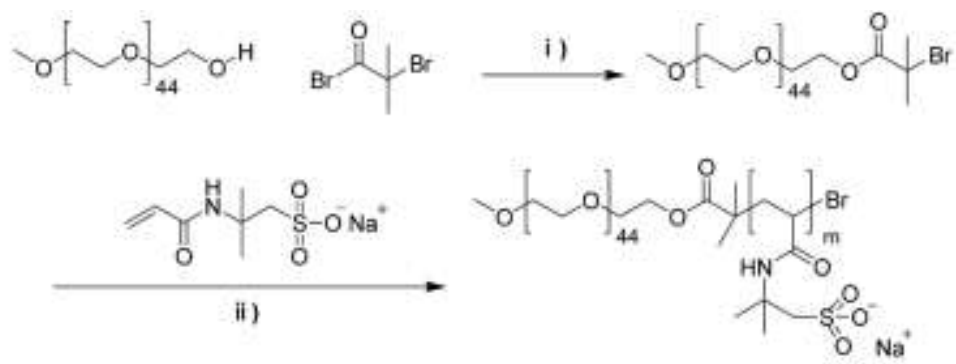

i) trimethylamine, THF; ii) 2,2'-bipyridine, $\mathrm{Cu}$ ( I) $\mathrm{Cl}, \mathrm{H} 2 \mathrm{O}, \mathrm{MeOH}$
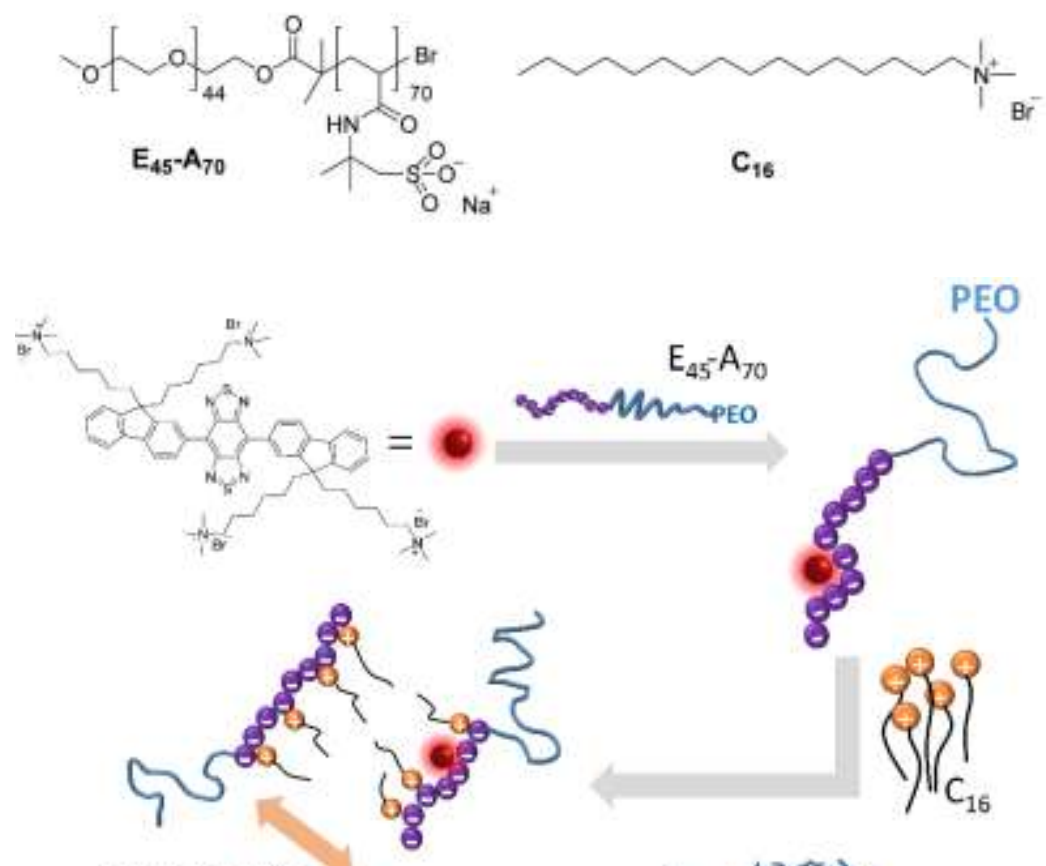

Hydrophobic

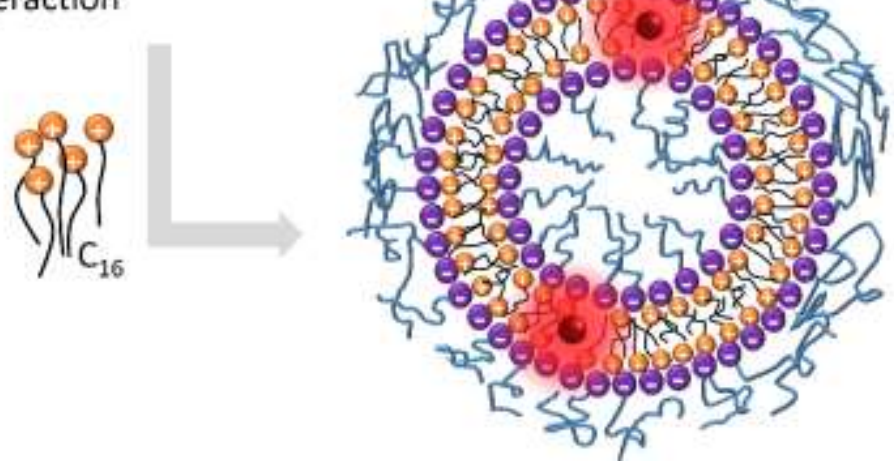

Scheme S1. Synthetic routes to $\mathbf{E}_{\mathbf{4 5}}-\mathbf{A}_{\mathbf{7 0}}$ and schematic for formation of COE/v. 

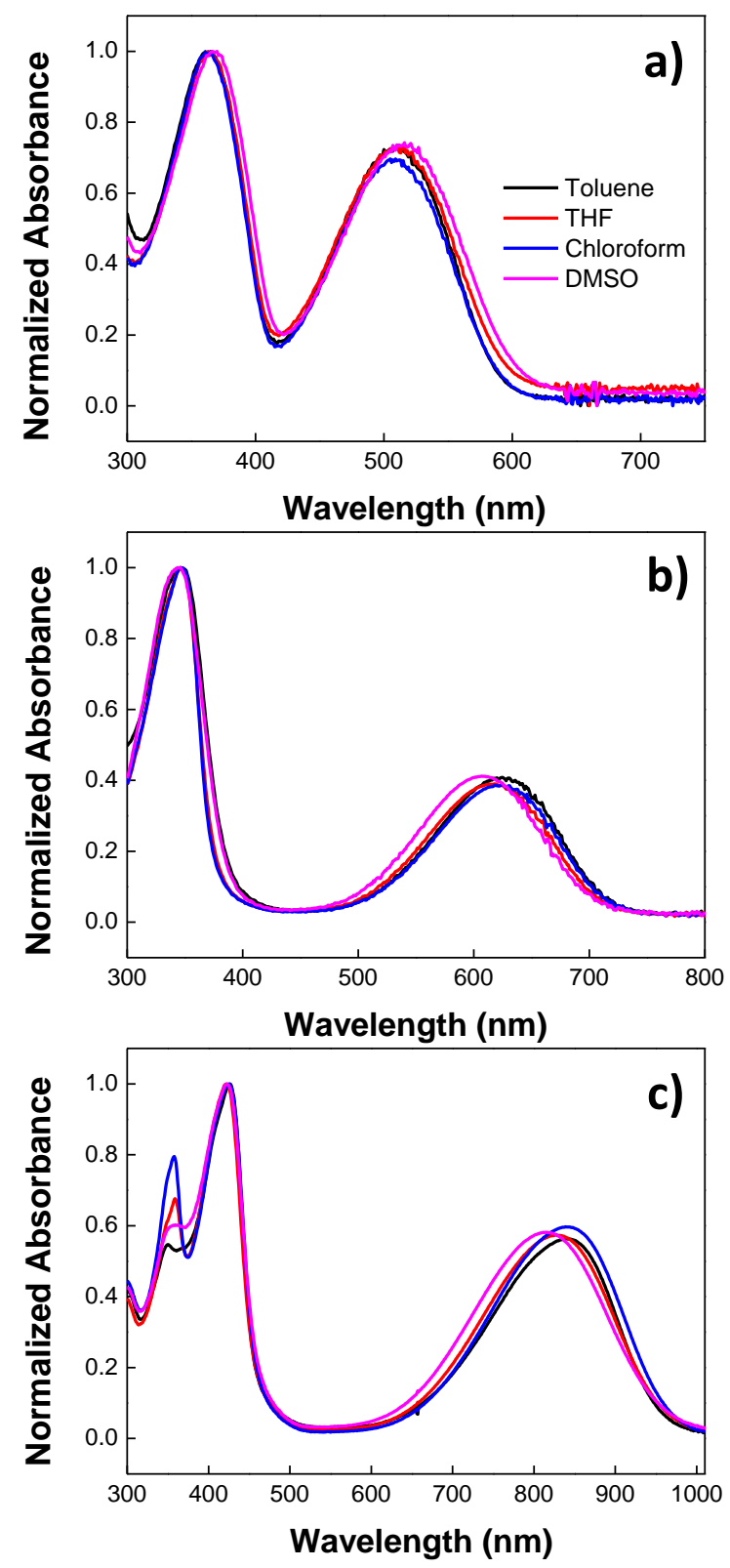

Figure S1. Normalized absorption spectra of neutral precursors, a) N-FITBTTFl, b) N-FIBBTFl and c) N-FITBBTTFl in various solvents. 

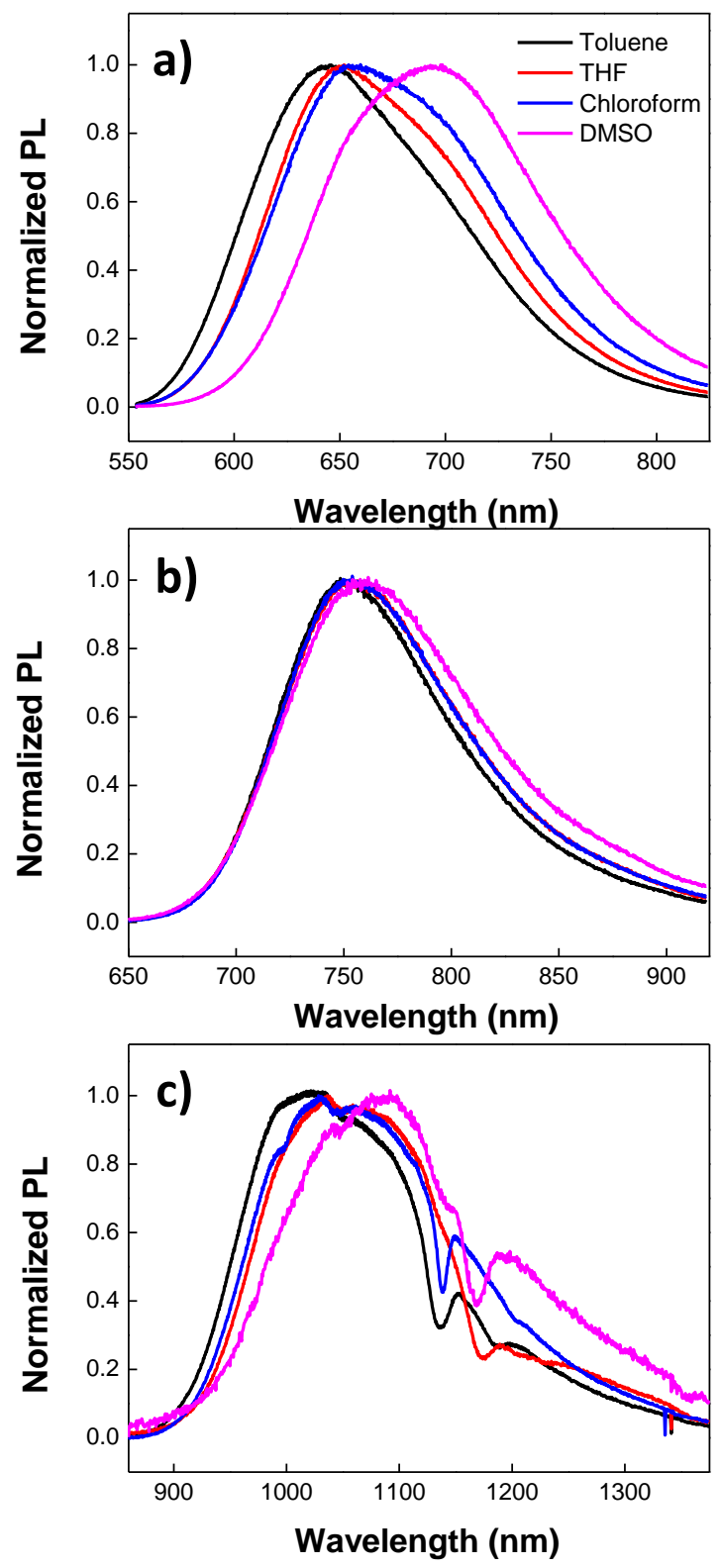

Figure S2. Normalized PL spectra of neutral precursors, a) N-FITBTTFI, b) N-FIBBTFl and c) N-FITBBTTFl in various solvents. 
Table S1. Summary of optical properties of neutral precursors in different solvents.

\begin{tabular}{ccccc}
\hline & Toluene & THF & Chloroform & DMSO \\
& $\lambda_{\text {abs }} / \lambda_{\mathrm{PL}}(\mathrm{nm})$ & $\lambda_{\mathrm{abs}} / \lambda_{\mathrm{PL}}(\mathrm{nm})$ & $\lambda_{\mathrm{abs}} / \lambda_{\mathrm{PL}}(\mathrm{nm})$ & $\lambda_{\mathrm{abs}} / \lambda_{\mathrm{PL}}(\mathrm{nm})$ \\
\hline N-FITBTTFI & $366,509 / 646$ & $363,514 / 652$ & $365,509 / 654$ & $370,516 / 698$ \\
& & & & \\
N-FIBBTFI & $347,625 / 751$ & $346,618 / 753$ & $346,625 / 753$ & $346,607 / 760$ \\
& & & & \\
N-FITBBTTFI & $426,840 / 1026$ & $422,827 / 1036$ & $425,839 / 1028$ & $423,815 / 1086$ \\
\hline
\end{tabular}
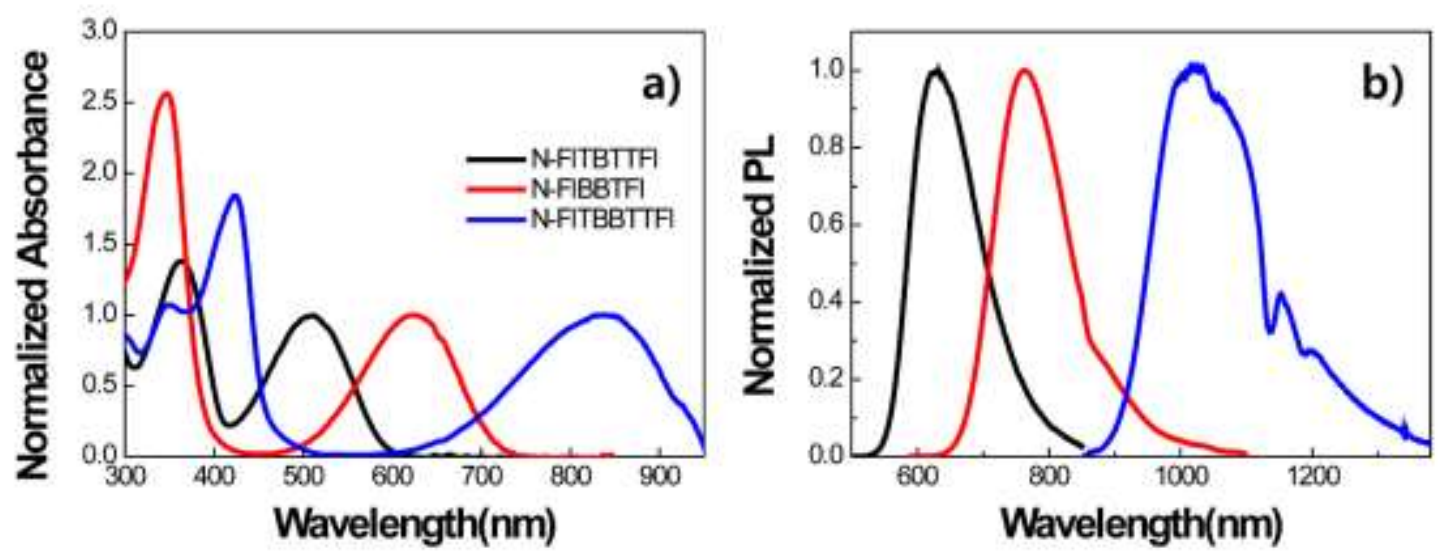

Figure S3. a) Normalized UV-vis and b) PL spectra of neutral NIR precursors in toluene. 


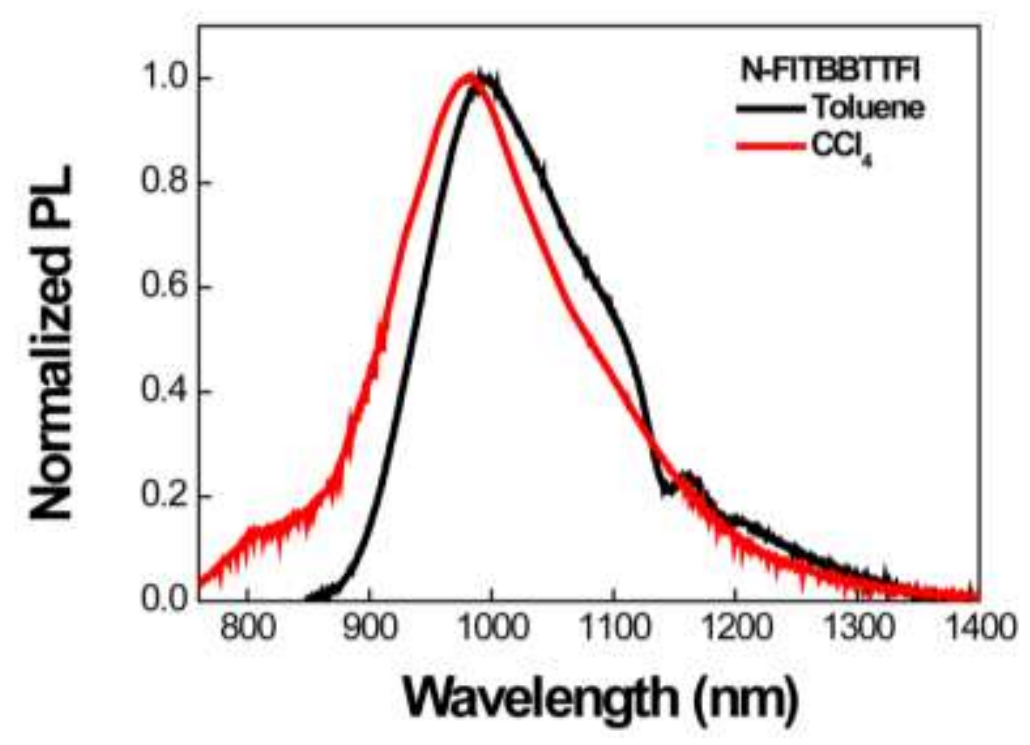

Figure S4. Normalized PL spectra of N-FITBBTTFl in toluene and in $\mathrm{CCl}_{4}$. 
Table S2. Summary of optical properties of NIR COEs before and after vesicular complex formation in water.

\begin{tabular}{lllll}
\hline & $\lambda_{\text {abs }}(\mathrm{nm})$ & $\lambda_{\mathrm{PL}}(\mathrm{nm})$ & $\Phi_{\mathrm{PL}}(\%)$ & Stokes shift $(\mathrm{nm})$ \\
\hline Q-FITBTTFI & 368,510 & 657 & 5.7 & 147 \\
Q-FITBTTFI/v & 370,526 & 659 & 27.8 & 133 \\
Q-FIBBTFI & 341,596 & 780 & 3.3 & 184 \\
Q-FIBBTFI/v & 345,632 & 770 & 13.4 & 138 \\
Q-FITBBTTFI & 422,814 & $\sim 1090$ & $<0.1$ & $\sim 276$ \\
Q-FITBBTTFI/v & 427,837 & $\sim 1050$ & 1.2 & $\sim 213$ \\
\hline
\end{tabular}

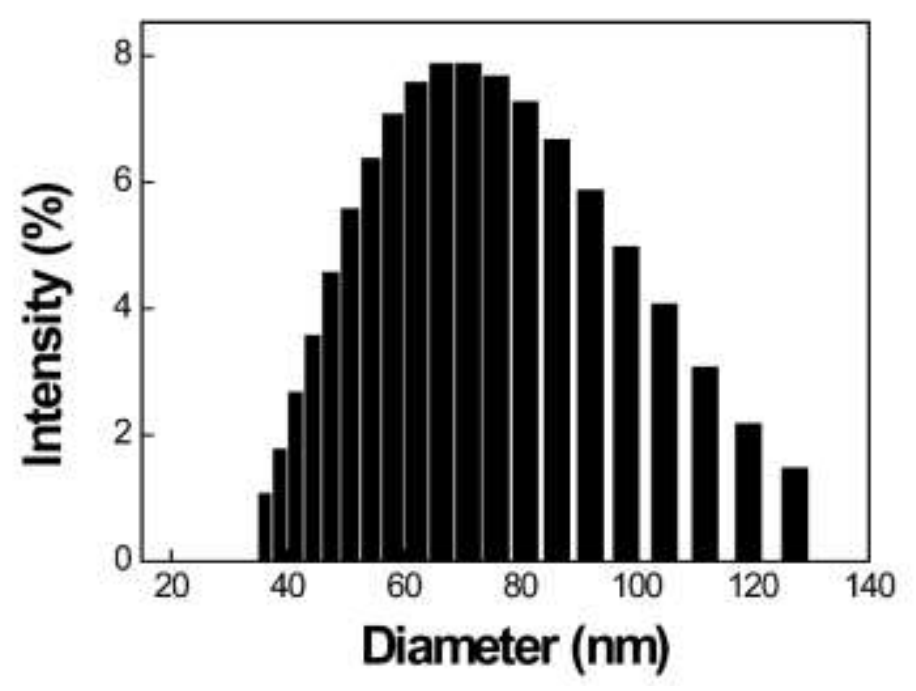

Figure S5. Dynamic light scattering data of a COE/v complex. 

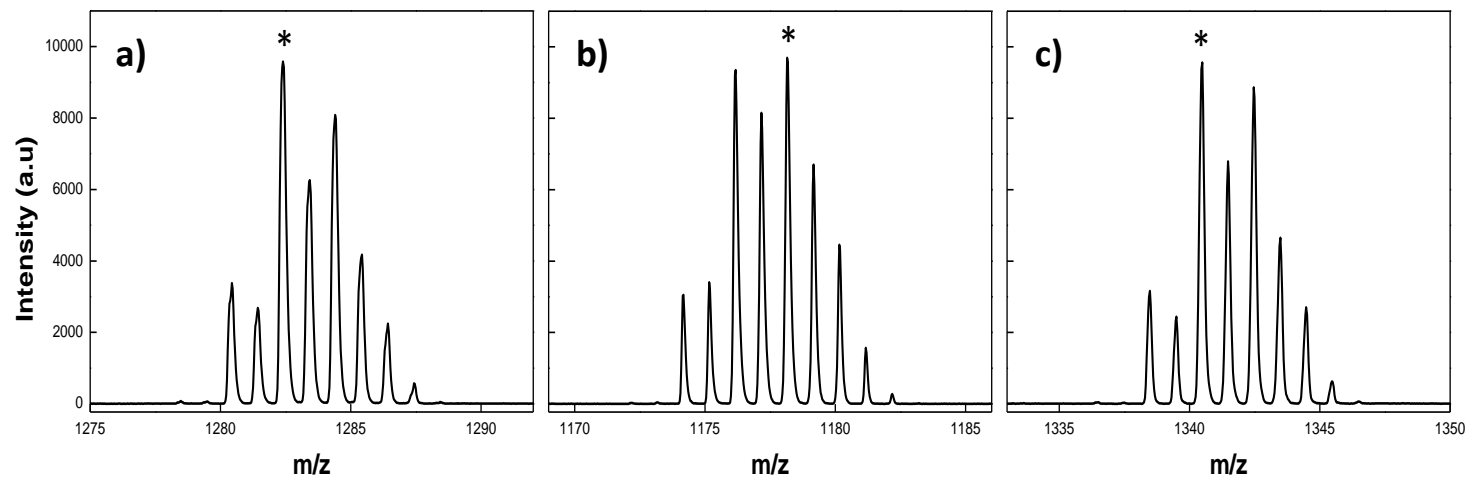

Figure S6. MALDI-TOF spectra of a) N-FITBTTFl, b) N-FIBBTFl, and c) N-FITBBTTFl. 


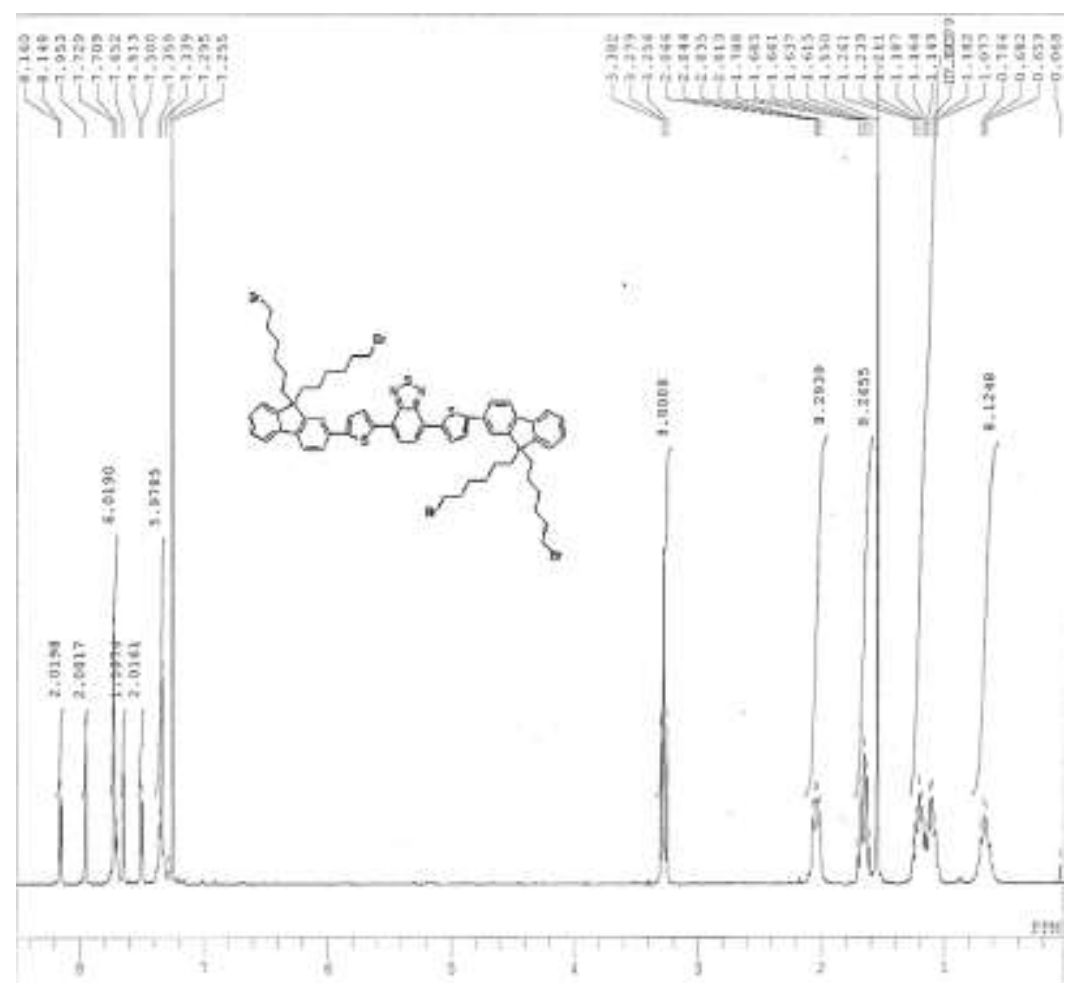

Figure S7. H NMR spectrum of N-FITBTTFl.

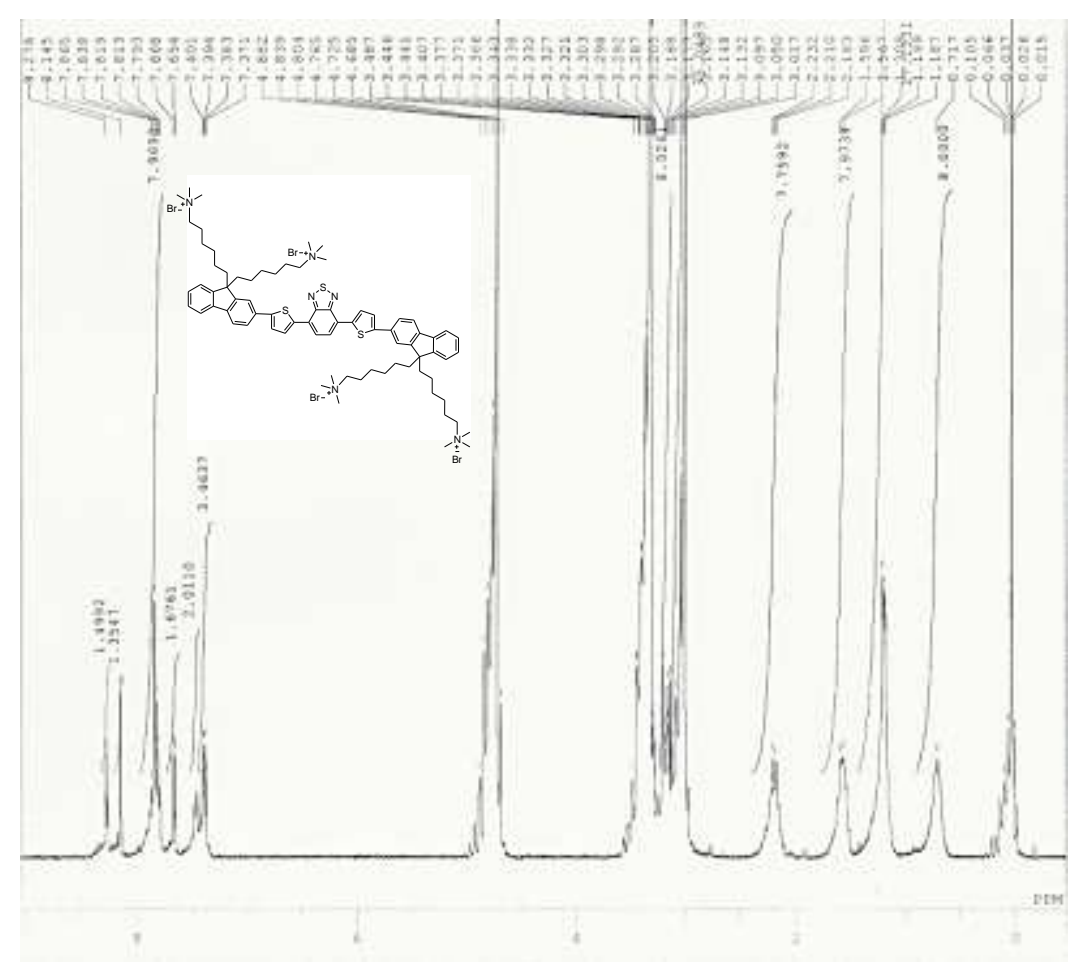

Figure S8. H NMR spectrum of Q-FITBTTFl. 


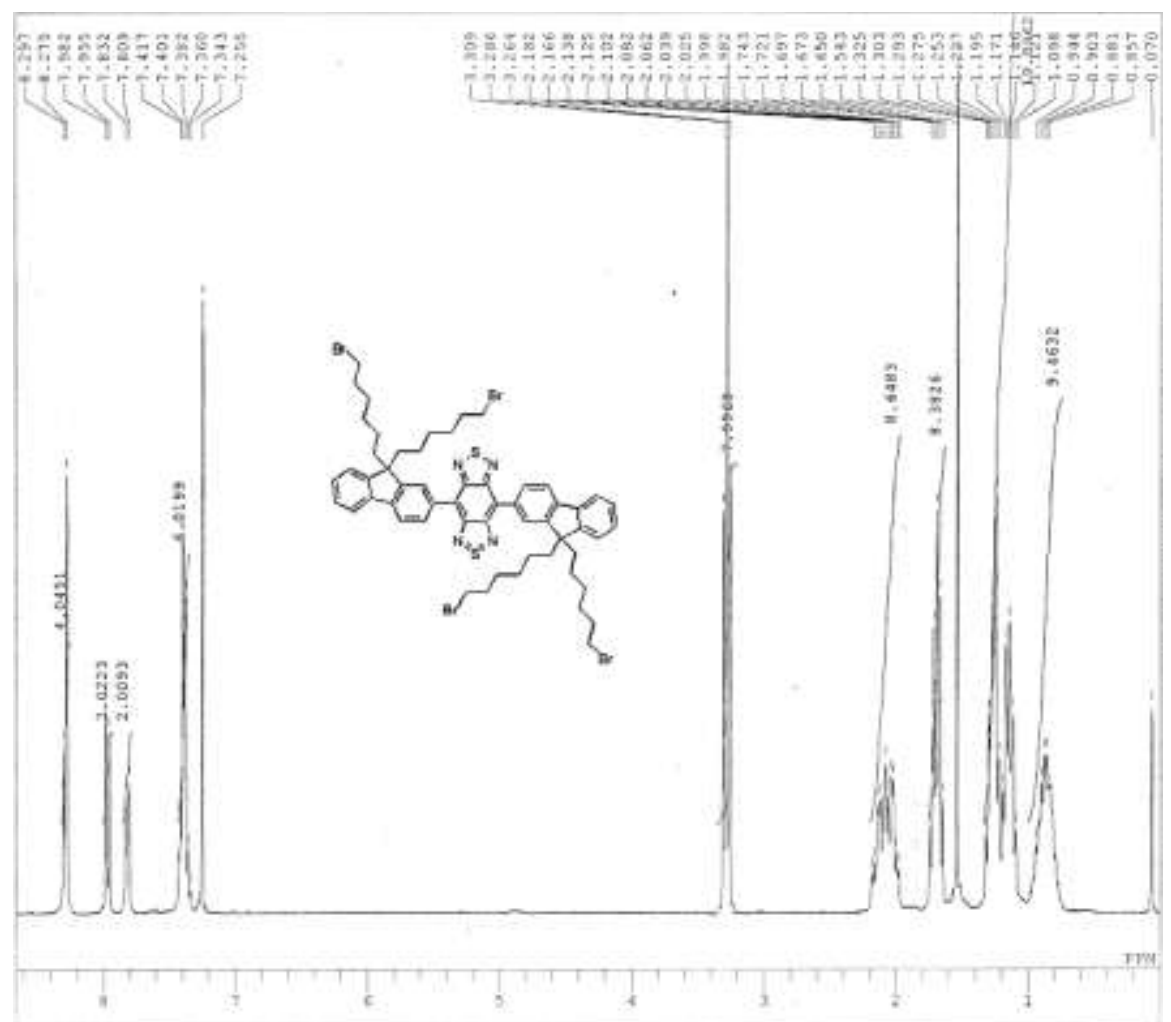

Figure S9. H NMR spectrum of N-FIBBTFI.

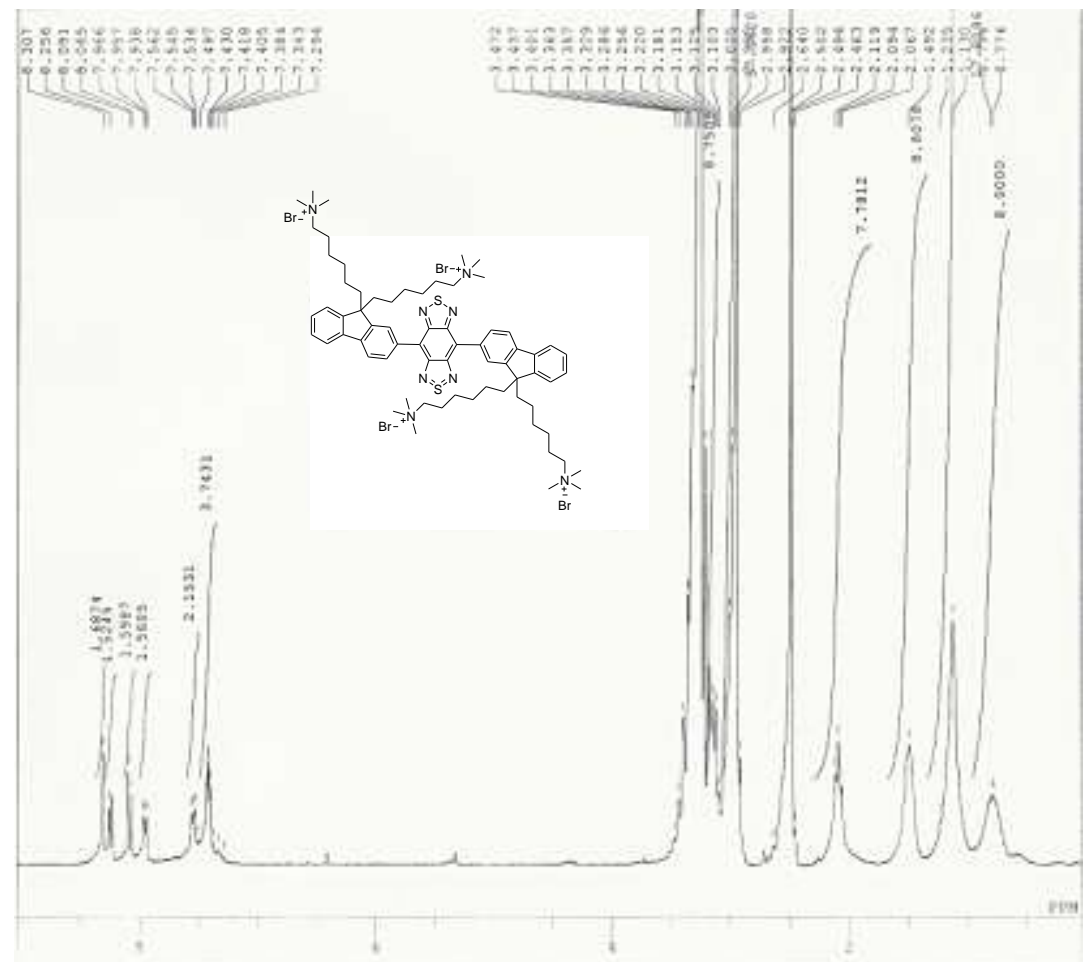

Figure S10. H NMR spectrum of Q-FlBBTFl. 


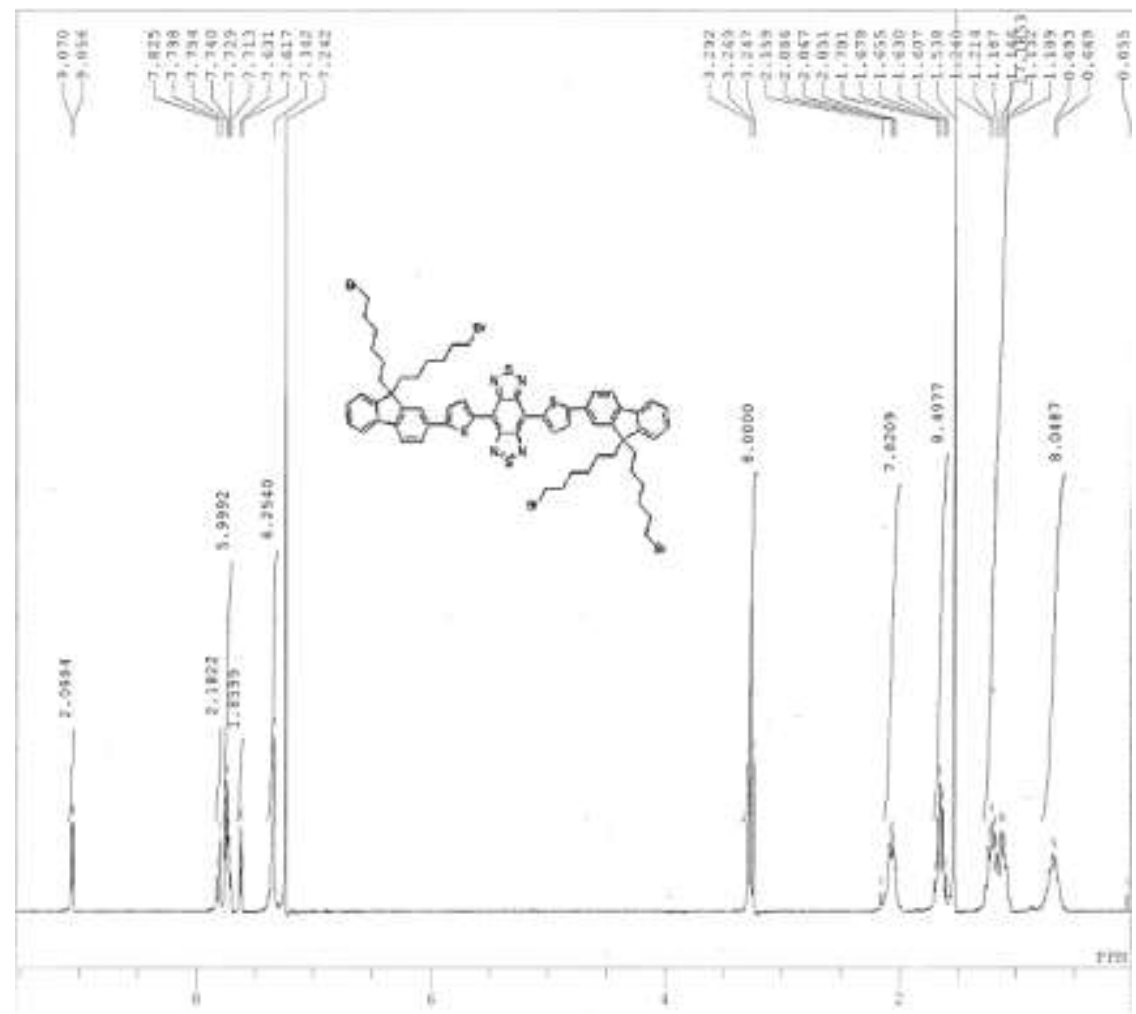

Figure S11. H NMR spectrum of N-FITBBTTFl.

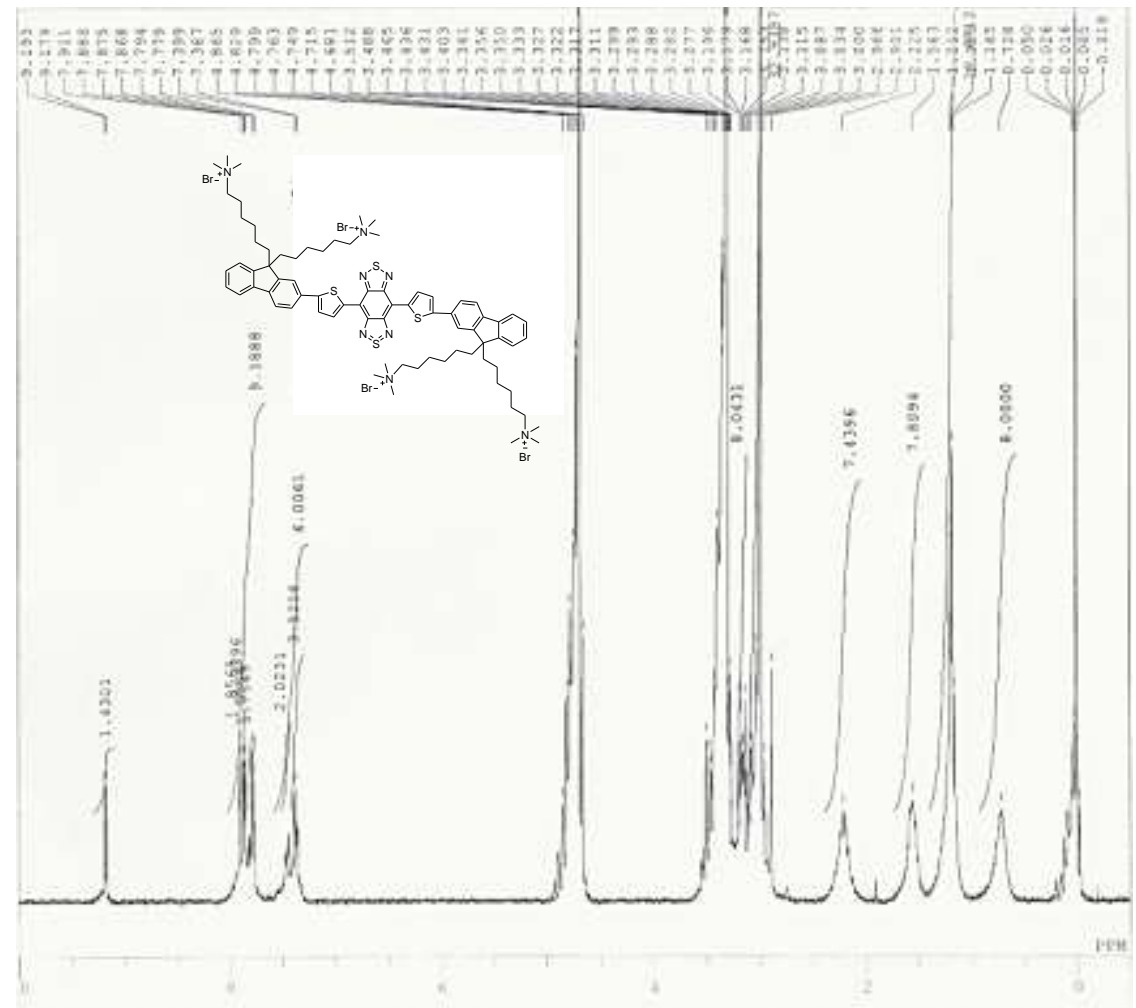

Figure S12. H NMR spectrum of Q-FITBBTTFl. 